TITLE:

\title{
Direct numerical simulation of a high-Froude-number turbulent open-channel flow
}

$\operatorname{AUTHOR}(S)$ :

Yamamoto, Yoshinobu; Kunugi, Tomoaki

\section{CITATION:}

Yamamoto, Yoshinobu ...[et al]. Direct numerical simulation of a high-Froude-number turbulent open-channel flow. PHYSICS OF FLUIDS 2011, 23(12): 125108.

\section{ISSUE DATE:}

2011-12

URL:

http://hdl.handle.net/2433/160673

\section{RIGHT:}

Copyright 2011 American Institute of Physics. This article may be downloaded for personal use only. Any other use requires prior permission of the author and the American Institute of Physics. The following article appeared in PHYSICS OF FLUIDS 23, 125108 (2011) and may be found at http://link.aip.org/link/?phf/23/125108 


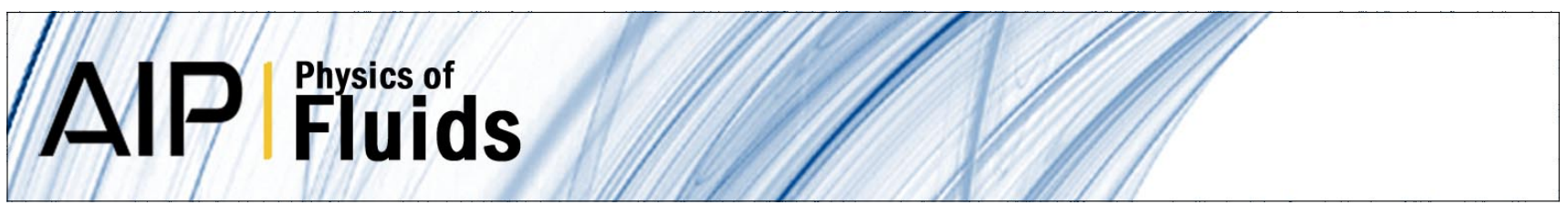

\section{Direct numerical simulation of a high-Froude-number turbulent open- channel flow}

Yoshinobu Yamamoto and Tomoaki Kunugi

Citation: Phys. Fluids 23, 125108 (2011); doi: 10.1063/1.3664121

View online: http://dx.doi.org/10.1063/1.3664121

View Table of Contents: http://pof.aip.org/resource/1/PHFLE6/v23/i12

Published by the American Institute of Physics.

\section{Related Articles}

Asymptotic expansion of the solution of the steady Stokes equation with variable viscosity in a two-dimensional tube structure

J. Math. Phys. 53, 103702 (2012)

Large-eddy simulation of turbulent channel flow using explicit filtering and dynamic mixed models

Phys. Fluids 24, 085105 (2012)

Anisotropy in pair dispersion of inertial particles in turbulent channel flow

Phys. Fluids 24, 073305 (2012)

About turbulence statistics in the outer part of a boundary layer developing over two-dimensional surface roughness

Phys. Fluids 24, 075112 (2012)

Accounting for uncertainty in the analysis of overlap layer mean velocity models

Phys. Fluids 24, 075108 (2012)

\section{Additional information on Phys. Fluids}

Journal Homepage: http://pof.aip.org/

Journal Information: http://pof.aip.org/about/about_the_journal

Top downloads: http://pof.aip.org/features/most_downloaded

Information for Authors: http://pof.aip.org/authors

\section{ADVERTISEMENT}

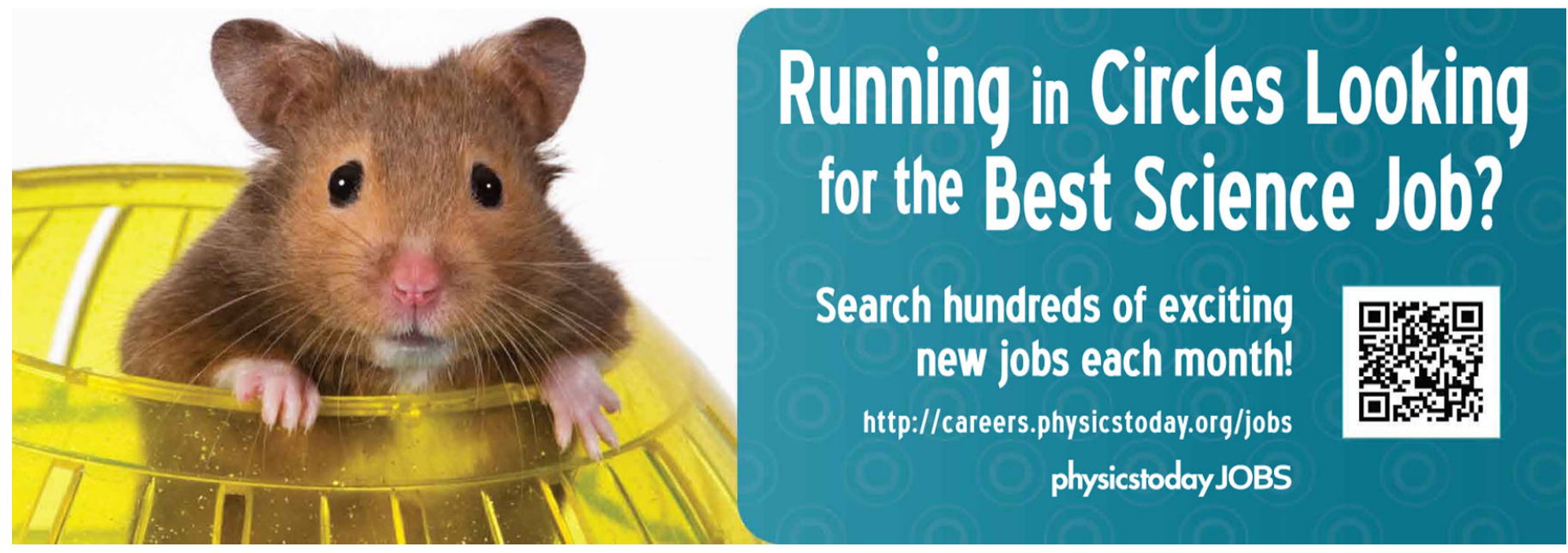




\title{
Direct numerical simulation of a high-Froude-number turbulent open-channel flow
}

\author{
Yoshinobu Yamamoto and Tomoaki Kunugi \\ Department of Nuclear engineering, Kyoto University, Yoshida Sakyo, Kyoto 606-8501, Japan
}

(Received 29 December 2010; accepted 7 September 2011; published online 16 December 2011)

\begin{abstract}
In this study, we successfully conducted a direct numerical simulation of a high-Fr turbulent open-channel flow at a Froude number of 1.8 and a Reynolds number of 2325 based on bulk velocity, gravitational acceleration, water depth, and kinetic viscosity, using the multi-interface advection and reconstruction solver (MARS). We confirmed that typical wall-bounded turbulent structures were observed in this high-Fr open-channel flow. On the other hand, near free-surface, surface deformations are constituted of large-scale gentle bumpy waves with the maximum wave height corresponding to approximately $4 \%$ of the water depth and small-scale isotropic waves on the large-scale waves. The large-scale waves would generate the high-speed streaky structures near the free-surface and the scale of the small waves would be equivalent to the near free-surface turbulent kinetic energy. Near the free-surface, wall-normal turbulent intensity and energy-dissipation rates increase toward the free-surface, and the tendencies of turbulent statistical quantities near the free-surface are in good agreement with the experimental measurements obtained using a laser Doppler anemometer (LDA) (Nakayama and Nezu, "Bursts near the free-surface in open-channel flows and their relationships with turbulence structures," J. Hydraulic, Coastal and Environmental Eng. JSCE 635/II, 31 1999). As well as the rigid-lid turbulent open-channel flow, turbulent intensity distribution from the vertical component to the streamwise one is predominant beneath the free-surface.
\end{abstract}

(c) 2011 American Institute of Physics. [doi:10.1063/1.3664121]

\section{INTRODUCTION}

Free-surface turbulence has been extensively investigated in various experiments. ${ }^{1-8}$ In open-channel turbulent flows, which may be imaged as river flows or liquid-film flows, the Froude number $\left[\mathrm{Fr}=U /(g h)^{1 / 2}\right.$, where $U$ is bulk mean velocity, $g$ is gravitational acceleration, and $h$ is flow depth] is an important factor regarding the effects of free-surface deformation on turbulent structures. Surface deformation is small in low-Fr range $(\mathrm{Fr}<0.5)$, hence free-surface can be regarded as a rigidlid free-surface. Therefore, the rigid-lid free-surface acts like a wall and restrains the surface-normal velocity component. ${ }^{3,5}$ Moreover, turbulent structures near the free-surface are closely related $^{1-7}$ to coherent vortices: surface-renewal eddies.

On the other hand, in high-Fr range ( $\mathrm{Fr}>1.0)$, it was found, ${ }^{5,8}$ using a laser Doppler anemometer (LDA), that the surface-normal turbulent intensity had a tendency to increase. Surface deformation effects on the wall-normal turbulent intensities could no longer be neglected; this indicated that turbulent structures that are different from those with a low-Fr flow would be expected in a high-Fr flow.

To understand coherent structures such as vortex and high-ordered turbulent statistics (e.g., energy-dissipation rate and pressure-strain correlations), direct numerical simulation (DNS) has advantages over experimental approaches. DNS of free-surface turbulent flow was initially conducted by Lam and Banerjee; ${ }^{9}$ they discussed the effect of interfacial shear stress on the formation of turbulent streaky structures near the free-surface. Handler et al. ${ }^{10}$ also conducted DNS of an openchannel flow and obtained turbulent statistics such as the budgets of Reynolds stresses near the rigid-lid free-surface. They pointed out that the pressure-strain correlation was dominant for the spanwise turbulent intensities and the dissipation rates for the horizontal components were reduced near the free-surface. Furthermore, many DNS (Refs. 11-21) were conducted to investigate the turbulent statistics and dynamical processes near the free-surface and so on. However, until now, all DNS (Refs. 9-21) of turbulent open-channel flows have been conducted under zero or low-Fr flow conditions. Some DNS that took into account surface deformations were performed by Komori et al., ${ }^{11}$ Borue et al., ${ }^{14}$ Thomas and Williams, ${ }^{15}$ Tsai, ${ }^{17}$ and Yokojima, ${ }^{21}$ but they demonstrated that the surface deformation effects on the free-surface turbulent structures were inactive; moreover, no increase in wallnormal turbulent intensity was observed under subcritical ( $\mathrm{Fr}<1)$ turbulent open-channel flow conditions.

The objective of this study is to perform a direct numerical simulation of super-critical turbulent open-channel flow at $\mathrm{Fr}=1.8$ by means of using the MARS (multi-interface advection and reconstruction solver) method ${ }^{22}$ for the first time. In this study, we report that the mean velocity, turbulent statistics, and surface fluctuations were investigated by comparing with those in low-Fr flow. The obtained database is of considerable value for studies of a turbulence model in super-critical turbulent open-channel flow.

\section{GOVERNING EQUATIONS AND NUMERICAL PROCEDURES}

\section{A. Continuity equation}

In the MARS method, ${ }^{22}$ transient spatial distributions of immiscible fluids consisting of gas and liquid, occupying at a certain point in the solution domain, can be represented by a 
volume fraction function $F$. The volume fraction function $F$ can be satisfied with the following relation:

$$
\sum F_{m}=1.0,
$$

where $F_{m}$ denotes the volume fraction of the $m$-th fluid. Then, for incompressible fluids under the assumption of a one-field model, ${ }^{23}$ the continuity equation can also be expressed as

$$
\frac{\partial F_{m}}{\partial t}+u_{i} \frac{\partial F_{m}}{\partial x_{i}}=\frac{\partial F_{m}}{\partial t}+\frac{\partial u_{i} F_{m}}{\partial x_{i}}-F_{m} \frac{\partial u_{i}}{\partial x_{i}}=0,
$$

where $u_{i}$ is the $i$ th component of the velocity $(i=1,2,3)$, $x_{1}(x)$ is the streamwise direction, $x_{2}(y)$ is the vertical direction, $x_{3}(z)$ is the spanwise direction, and $t$ is time.

\section{B. Momentum equation}

The momentum equations of incompressible and Newtonian fluids, using the CSF (continuum surface force) model, ${ }^{24}$ can be written in the form

$$
\frac{\partial u_{i}}{\partial t}+\frac{\partial u_{i} u_{j}}{x_{j}}=g \delta_{i 2}-\frac{1}{\langle\rho\rangle} \frac{\partial p}{\partial x_{i}}+\frac{\partial \tau_{i j}}{\partial x_{j}}+\frac{F v_{i}}{\langle\rho\rangle},
$$

where thermal properties at the interface, the viscous stress $\tau_{i j}$, and volume force according to the CSF model, $F v_{i}$, are defined by the following equations:

$$
\begin{aligned}
& \langle\rho\rangle=\sum F_{m} \rho_{m}, \\
& \bar{\rho}=\sum \rho_{m} / 2 \text {, } \\
& \tau_{i j}=\frac{\langle\langle\mu\rangle\rangle}{\langle\langle\rho\rangle\rangle}\left(\frac{\partial u_{i}}{\partial x_{j}}+\frac{\partial u_{j}}{\partial x_{i}}\right) \text {, } \\
& F v_{i}=\sigma \kappa \frac{\partial F_{l}}{\partial x_{i}} \frac{\langle\rho\rangle}{\bar{\rho}} \\
& \kappa=-\frac{\partial n_{i}}{\partial x_{i}} \\
& \left.\langle\langle\phi\rangle\rangle=\left[\phi_{l} \times\left\{\operatorname{sign}\left(F_{g}-F_{l}\right)+1\right\}+\phi_{g} \times\left\{1-\operatorname{sign}\left(F_{l}-F_{g}\right)\right\}\right] / 2\right\}
\end{aligned}
$$

where $g, \rho, \mu, p, \sigma, \kappa$, and $n_{i}$ denote gravitational acceleration, density, viscosity, pressure, coefficient of surface tension, interface curvature, and $i$ th components of the interface normal vector, respectively. Here, $\langle\langle\phi\rangle\rangle$ indicates that the thermal property at the interface is defined by the thermal property that has a greater $F$ value. Subscripts $g$ and $l$ denote air and water, respectively.

\section{Numerical procedures}

Regarding the discretization of the velocity fields on the Cartesian coordinate system, the second-order scheme for the spatial differencing terms is used on the staggered grid system. The time integration method is based on the fractional-step method ${ }^{25}$ and the third-order low-storage Runge-Kutta scheme is adapted for the convection terms, the Crank-Nicolson scheme is adapted for the viscous term, and the Euler implicit scheme for the pressure term. Fractional-step velocity $\left(u_{i}^{F}\right)$ is obtained by solving the momentum equations using the conjugate residual (CR) method, and the following Poisson equation is solved by the incomplete LU biconjugate gradient (ILUBCG) method:

$$
\alpha_{n} \Delta t \frac{\partial}{\partial x_{i}}\left(\frac{1}{\langle\rho\rangle} \frac{\partial \varphi}{\partial x_{i}}\right)=\frac{\partial u_{i}^{F}}{\partial x_{i}},
$$

where $\alpha_{n}\left(\alpha_{1}=4 / 15, \alpha_{2}=1 / 15\right.$, and $\left.\alpha_{3}=1 / 6\right)$ is the coefficient of the third-order low-storage Runge-Kutta scheme, $n$ is the Runge-Kutta substep rank, and the variable $\varphi$ is related to the pressure and is given by

$$
p=\varphi-\frac{\alpha_{i r k} \Delta t\langle\langle\mu\rangle\rangle}{2} \frac{\partial}{\partial x_{i}}\left(\frac{1}{\langle\rho\rangle} \frac{\partial \varphi}{\partial x_{i}}\right)
$$

Finally, the new time-step velocity can be obtained by

$$
u_{i}=u_{i}^{F}-\frac{\alpha_{i r k} \Delta t}{\langle\rho\rangle} \frac{\partial \varphi}{\partial x_{i}}
$$

The time discretized volume transport Eq. (2) can be expressed as

$$
F_{m}^{n+1}=F_{m}^{n}-\frac{\partial\left(\delta F_{m}\right)_{i}^{n+1}}{\partial x_{i}}-F_{m}^{n}\left(\frac{\partial u_{i}}{\partial x_{i}}\right)^{n+1} \alpha_{n} \Delta t
$$

where $\left(\delta F_{m}\right)_{i}$ is the $i$ th component of transport volume flux defined at the $i$ th component velocity point. This transport volume flux can be expressed as

$$
\left(\delta F_{m}\right)_{i}=\int_{x_{i}-u_{i} \Delta t}^{x_{i}} S_{i} d x_{i}
$$

where, $S_{i}$ is the fluid-area fraction as shown in Fig. 1. The detailed calculation procedure for the fluid-area fraction is described by Kunugi. ${ }^{22}$ Consequently, the velocity, pressure, and volume fraction at the new time-step can be obtained. 


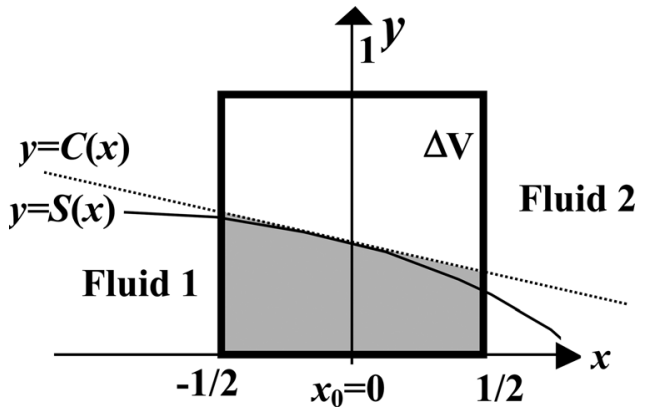

FIG. 1. Normalized computational cell, fluid-area fraction $S(x)$, and linesegment function $C(x)$.

\section{PROBLEM DESCRIPTIONS}

\section{A. High-Fr open-channel flow}

A schematic view of a high-Fr open-channel flow at $\mathrm{Fr}=1.8$ is shown in Fig. 2(a) and the numerical conditions are listed in Table I. Here, $\operatorname{Re}_{\tau}\left(=u_{\tau} h / \nu_{w}\right)$ is the turbulent Reynolds number based on friction velocity at the bottom wall $\left(u_{\tau}\right)$, the water depth (= air phase height $\left.h\right)$, and the kinetic viscosity of water $\left(\nu_{w}\right)$, where subscripts $g$ and $w$ denote air and water, respectively.

Under these DNS conditions, the Froude number was almost the same as that in a previous experimental study ${ }^{8}$ except for the fact that in that study, the Reynolds number was only $1 / 10$. In order to determine whether the effect of gravity exceeds the effect of surface tension on the freesurface turbulent structure, we used the surface tension coefficient on a scale of $1 / 10\left(\sigma=7.27 \times 10^{-3}[\mathrm{~N} / \mathrm{m}]\right)$. Consequently, the ratio between the Reynolds number and the Weber number (We $=\rho_{g} u_{\tau} h / \sigma$, where $\sigma$ is the surface tension coefficient) was the same as that in the previous experimental study. ${ }^{8}$ Thermal properties of water and air, except the surface tension coefficient, at room temperature were used. $L_{x}, L_{y}$, and $L_{z}$ are the computational domain lengths, $N_{x}, N_{y}$, and $N_{z}$ are the grid numbers, and $\Delta x^{+}, \Delta y^{+}$, and $\Delta z^{+}$are the grid sizes for streamwise $(x)$, vertical $(y)$, and spanwise $(z)$ directions, respectively. Superscript + denotes the dimensionless quantities normalized by the wall units based on the friction velocity and kinetic viscosity of water. Vertical grid resolution was able to resolve the Kolmogorov scale of the rigid-lid turbulent open-channel flow with the same Reynolds number, and the horizontal grid resolutions were set to 5 for the wall units. To examine the horizontal griddependency on the DNS results, a resolution approximately 2 times finer (case $1^{\prime}$ ) was also conducted.

\section{B. Rigid-lid open-channel flow and parallel channel flow}

DNS of two single-phase turbulent flows were conducted, compared to turbulent structures of the high-Fr case. One was a zero-Fr (e.g., rigid-lid) open-channel flow, as shown in Fig. 2(b), and the other was a parallel channel flow, as shown in Fig. 2(c). The Navier-Stokes equations for incompressible fluid and the continuity equation were also solved by the fractional-step method, ${ }^{25}$ and the third-order low-storage Runge-Kutta scheme and the Crank-Nicolson scheme were used for the convection and viscous terms, respectively. A Fourier-spectral method for the horizontal directions and the second-order finite-difference method in the wall-normal direction were adopted for spatial discretization. Aliasing errors for the horizontal directions were removed using the two-thirds rule. ${ }^{26}$ Grid arrangements for the streamwise and spanwise velocities were located at the pressure point, and the grid point of the wall-normal velocity was shifted a half-grid distance away from the pressure point.

\section{Initial and boundary conditions}

Regarding the initial conditions for the high-Fr openchannel flow, the water surface was set to be flat, the velocity fields obtained from the rigid-lid case (case 2) were applied to the velocity fields of water, and the air layer velocity was set to zero. Pertaining to the boundary conditions, periodic boundary conditions in the spanwise $(x)$ and streamwise $(z)$ directions were imposed; the no-slip wall condition was applied to both the upper boundary of air and the lower boundary of water. To maintain the initial momentum flux difference between air and water sides, a constant streamwise mass flux condition was imposed for both the water and air side. Consequently, the bulk Reynolds number in the water phase was 2325 in the high-Fr case. In the air-liquid two-phase flows with surface deformations, the definition of

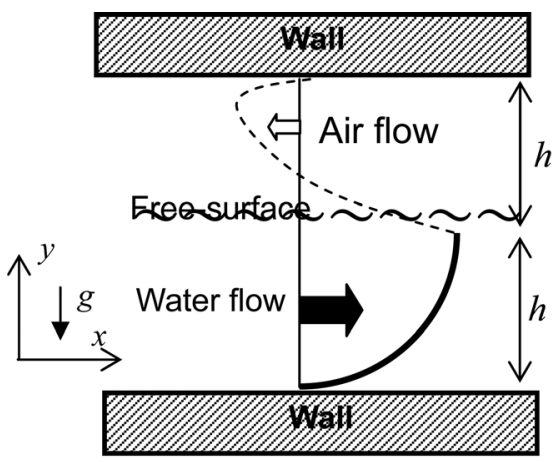

(a)

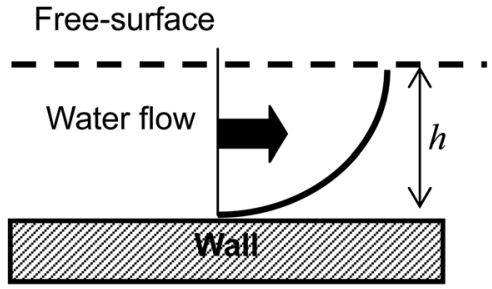

(b)

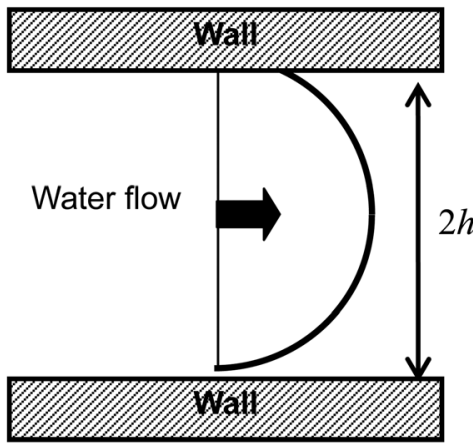

(c)

FIG. 2. Schematic views of the present flow fields: (a) high-Fr open-channel flow, (b) rigid-lid open-channel flow, and (c) parallel channel flow. 
TABLE I. Numerical condition: $\operatorname{Re}_{\tau}=u_{\tau} h / \nu_{w}$ : turbulent Reynolds number; $u_{\tau}$ : friction velocity at bottom wall; $h$ : water depth (=gas layer height); $\nu_{w}$ : kinetic viscosity of water, $\mathrm{Fr}=U_{m} /(g h)^{1 / 2}$ : Froude number; $U_{m}$ : bulk mean velocity at water side; $\rho_{w}$ : liquid density; $\rho_{g}$ : air density; $L_{x}, L_{y}, L_{z}$ : computational domain; $N_{x}, N_{y}, N_{z}$ : grid number; $\Delta x^{+}, \Delta y^{+}, \Delta z^{+}$: grid resolution for streamwise $(x)$, vertical $(y)$, and spanwise $(z)$ directions, respectively. Superscript + denotes the dimensionless quantities normalized by friction velocity and kinetic viscosity using the definition of the Reynolds number. $T_{0}{ }^{+}$: time integration length from initial condition to fully developed status; $T^{+}$: time integration length after fully developed status.

\begin{tabular}{|c|c|c|c|c|c|c|c|c|}
\hline & $\operatorname{Re}_{\tau}$ & $\mathrm{Fr}$ & $\rho_{\mathbf{w}} / \rho_{\mathbf{g}}$ & Domain $L_{x} \times L_{y} \times L_{z}$ & Grid number $N_{x} \times N_{y} \times N_{z}$ & Resolution $\Delta x^{+}, \Delta y^{+}, \Delta z^{+}$ & $T_{0}^{+}$ & $T^{+}$ \\
\hline Case1 & 150 & 1.8 & 842.1 & $12.8 h \times 2 h \times 6.4 h$ & $384 \times 214 \times 192$ & $5.0,0.26-2.0,5.0$ & 610 & 730 \\
\hline Case $1^{\prime}$ & 150 & 1.8 & 842.1 & $12.8 h \times 2 h \times 6.4 h$ & $648 \times 214 \times 324$ & $3.0,0.26-2.0,3.0$ & 610 & 730 \\
\hline Case2 & 150 & 0 & - & $12.8 h \times 2 h \times 6.4 h$ & $384 \times 107 \times 192$ & $5.0,0.26-2.0,5.0$ & - & 700 \\
\hline Case 3 & 150 & - & - & $12.8 h \times 2 h \times 6.4 h$ & $384 \times 182 \times 192$ & $5.0,0.26-2.0,5.0$ & - & 700 \\
\hline
\end{tabular}

fully developed status is vague to some extent. In this study, the time-entrance length $\left(T_{0}{ }^{+}\right)$was 610 , and mean and statistical values were obtained by time and horizontal plane averaging for 730 dimensionless time units $\left(T^{+}\right)$after $T_{0}{ }^{+}$. This time integration length corresponds to the fetch length equivalent to 75 times the water depth. During this time integration, the intensities of free-surface deformation did not show the monotonically increasing or decreasing tendency as shown in Fig. 3(a), and the flow fields can be regarded as reaching a statistically pseudo-steady status.

\section{RESULTS AND DISCUSSIONS}

\section{A. Free-surface structure}

Figures 3(a) and 3(b) show the root-mean-square (R.M.S.) of $F$ in the high-Fr open-channel flow (case 1). Figure 3(a) indicates that the temporal maximum (or minimum) water height changed by 3 or 4 wall units. The averaged surface intensity profile is almost symmetrical, and the
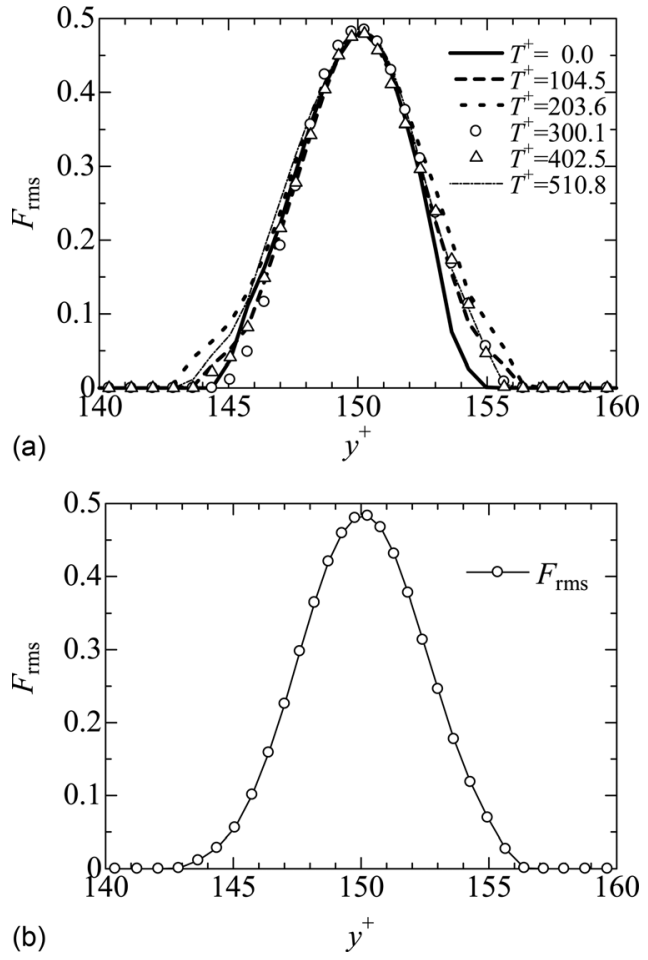

FIG. 3. R.M.S. of VOF function profiles, case 1: profiles of time history of R.M.S. of $F$ and (b) profile of averaged R.M.S. of $F$. maximum wave height, which was resolved on 20 grids in the $y$-direction, approximately corresponds to $4 \%$ of the water depth. These results indicated that the surface deformations in this high-Fr turbulent open-channel flow consist of the large-scale waves and small-scale waves on them, and the averaged large-scale waves was symmetric about the initial water height $\left(y^{+}=150\right)$.

Figure 4(a) shows instantaneous water surfaces, in which water surface defined as the iso-surface of $F$ is 0.5 . Waves with high wave numbers were observed on largescale waves with gentle bumps. The distribution of watersurface height in the streamwise direction at $z^{+}=100$ is shown in Fig. 4(b). Four or five large-scale waves and some small-scale waves were observed.

\section{B. Grid dependency on total mean and statistics in high-Fr flow}

Figures 5 and 6 show the mean velocity and the turbulent intensity profiles in the high-Fr flows (case 1 and case $\left.1^{\prime}\right)$. Note that these mean velocity and turbulent intensities were averaged without distinguishing between air and water phases. As shown in Fig. 5, air and water flow in a countercurrent direction with respect to each other, except for the air flow near the free-surface. The mean velocity profile of the air flow shows a parabolic shape. This indicates that the air flow is a laminar flow and not a turbulent one. Turbulent intensity profiles, as shown in Fig. 6, exhibit peaks at the freesurface caused by the surface deformation. Despite the difference in the horizontal grid resolutions between case 1 and case $1^{\prime}$, both mean velocity and turbulent intensities in case 1 and case $1^{\prime}$ are in good agreement with each other. Therefore, the results of case 1 are hereafter used as the data in the high-Fr case.

\section{Visualizations of near wall turbulent structures and interaction between air and water phase in high-Fr flow}

Instantaneous streamwise velocity contours and schematic description of streamwise velocity in case 1 (high-Fr case) are shown in Figs. 7(a) (side view) and 7(b) (end view), respectively. On the water side, typical turbulent shear flows, composed of upwelling or surface-renewal vortices, ${ }^{2-7,9-11,13,16-21}$ are observed along with the DNS results $^{11,13,19}$ of the low-Fr open-channel flow. Streamwise length of the upwelling was visually approximated as $2 h$ ( $=300$ in wall units). 


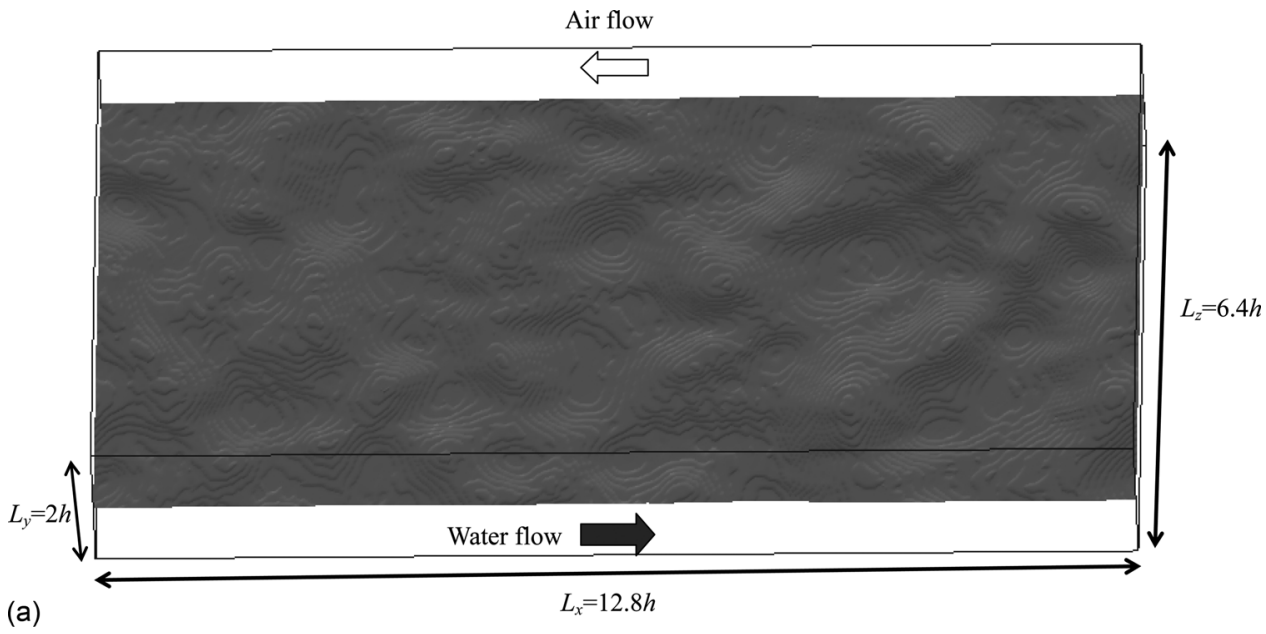

FIG. 4. Instantaneous free-surface characteristics, case 1: (a) free-surface distribution (iso-surface of $F=0.5$ ), bird's eye view, and (b) profile of watersurface height for streamwise direction at $z^{+}=100$.

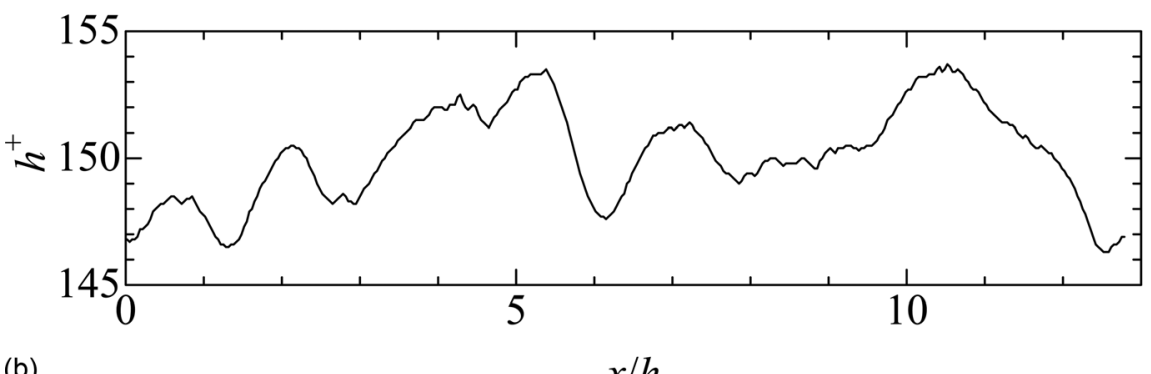

(b)

$x / h$

On the other hand, the velocity ranges on the air side were almost constant compared to the water side expect for near the free-surface. These results indicated that air-liquid interface interaction was inactive, and the present flow field in case 1 can be regarded as a true "open-channel flow." Schematic descriptions of near-wall coherent structures in Figures 7(a) and 7(b) were shown in them. As the results, we can confirm that near wall structures were as same as those usually observed in channel or boundary layer flow and the effects of interactions between air and liquid phase on turbulent generations would be indifference.

\section{Visualizations of near free-surface turbulent structures in high-Fr flow}

Figures 8 (a) and 8 (b) show the streamwise turbulent velocity distributions in the high-Fr case. Note that Fig. 8(a) near the free-surface $\left(y^{+}=138.5\right)$ and Fig. $8(\mathrm{~b})$ at the freesurface $\left(y^{+}=149.7\right)$ regions. Streamwise high-speed streaky and low-speed spotty structures can be observed in both Figs. 8(a) and 8(b). Figure 8(c) shows the volume of fluid (VOF) function distribution at wall-normal height $y^{+}=149.7$. In this wall-normal height, both air and water phases were present and the areas indicated in white and black are corresponded to the air-phase and the water-phase, respectively. As contrasted with Figs. 8(b) and 8(c), it can be understood that the low-speed spotty areas in Fig. 8(b) were caused by the valley positions of the surface waves in Fig. 8(c). In Fig. 8(a), there is no air-phase area at the wallnormal height of $y^{+}=138.5$ as shown in Fig. 3. Nevertheless, the low-speed spotty areas were also related with the valley positions of them.

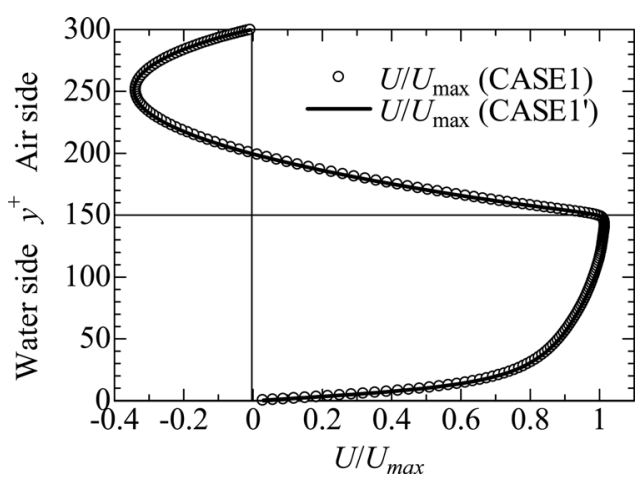

FIG. 5. Mean velocity profiles, $\mathrm{Fr}=1.8\left(\right.$ case 1 and case $\left.1^{\prime}\right)$.

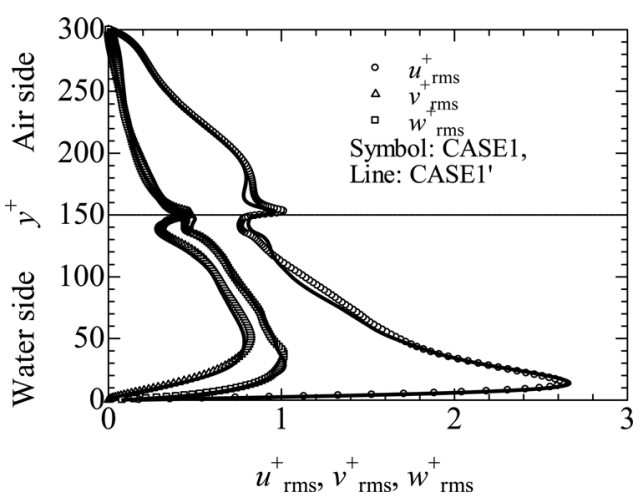

FIG. 6. Turbulent intensity profiles, $\mathrm{F}=1.8$ (case 1 and case $1^{\prime}$ ). 


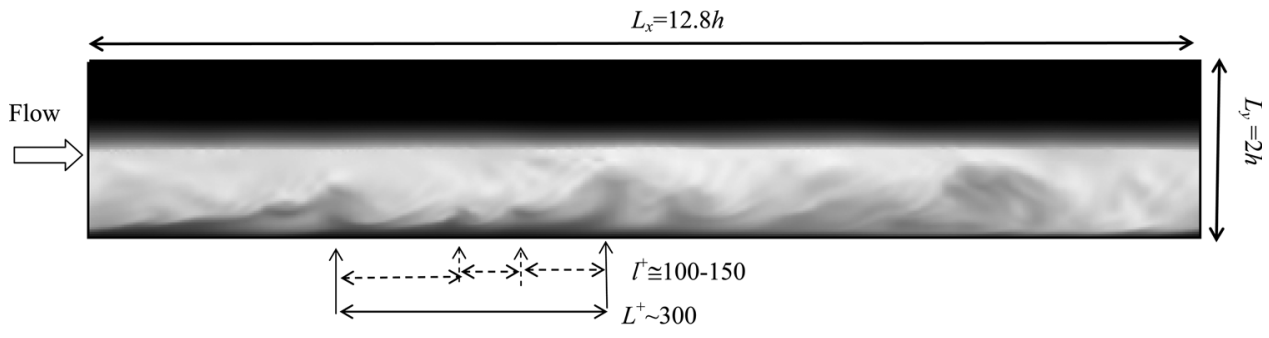

Side view, 0.0 (black) $<U^{+}+u^{+}<20.1$ (white),

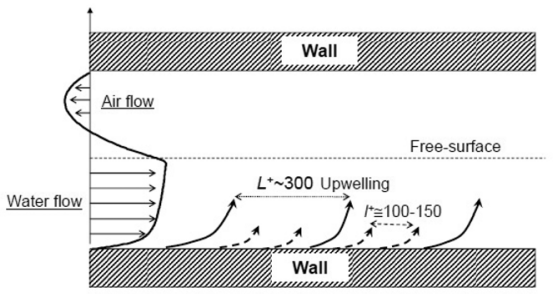

(a)

High-Fr flow, side view

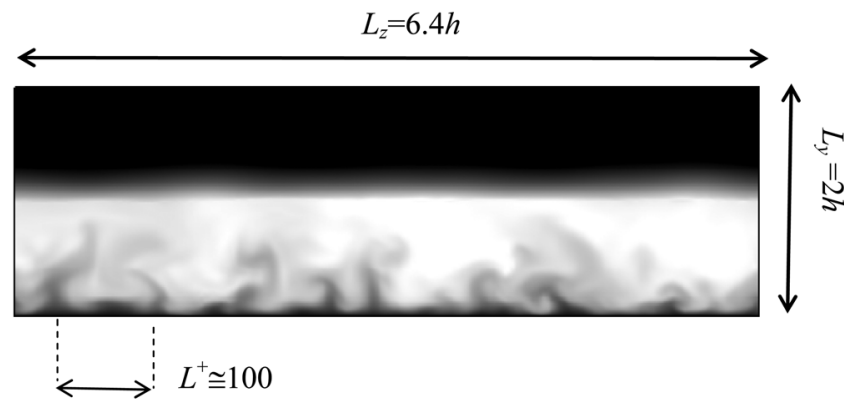

FIG. 7. Contour plots of instantaneous streamwise velocity and schematic description, case 1: (a) side view and (b) end view.

End view, contour plots, 0.0 (black) $<U^{+}+u^{+}<18.7$ (white)

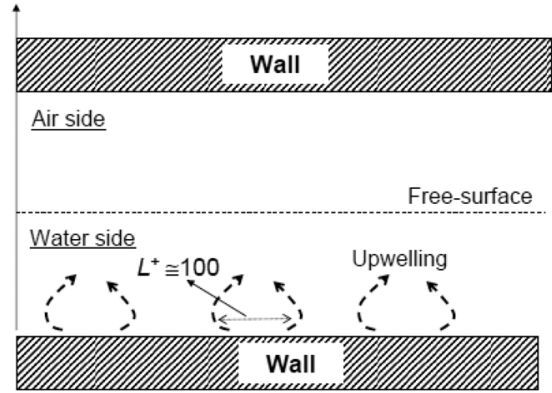

(b)

High-Fr flow, end view

The high-speed streaky structures (indicated in white) were also observed in both Figs. 8(a) and 8(b) and these structures can be correlated with each other. For generation of the streaky structures, Lee et al. ${ }^{27}$ concluded that high shear rate is sufficient, however there was less mean velocity gradient in water phase as discussed in session E (Fig. 10). The reasons are why the main stream flow was blocked by the free-surface dip and went around the side of one or plunged below one. That is to say, the large-scale gentle bumpy waves might play the same role in roughness. Therefore, only the high-speed streaky structures were observed but the low-speed streaks cannot be appeared near the free-surface. This conceptual sketch of the high-speed streaks and the low-speed sports near the free-surface is drawn in Fig. 8(d).

Figure 8(e) shows the vertical turbulent velocity distributions at the wall-normal height $y^{+}=149.7$. High wave- number streamwise and vertical velocity fluctuations show the strong associated with the surface deformations as shown in Fig. 4(a).

\section{E. Spectral analysis near free-surface in high-Fr flow}

Figures 9(a)-9(c) show the energy spectrum profiles of turbulent velocity components in the high-Fr case. Note that Fig. 9(a) is near the water-bottom wall and Figs. 9(b) and 9(c) are near the free-surface. The effect of surface deformations on the velocity fluctuations cannot be observed near the water-bottom wall; whereas, it is evident in all components of the streamwise energy spectrum in the regions with high wave numbers near the free-surface. In the near free-surface spanwise energy spectrum, as shown in Fig. 9(c), the spanwise and wall-normal components exceed the streamwise ones. 


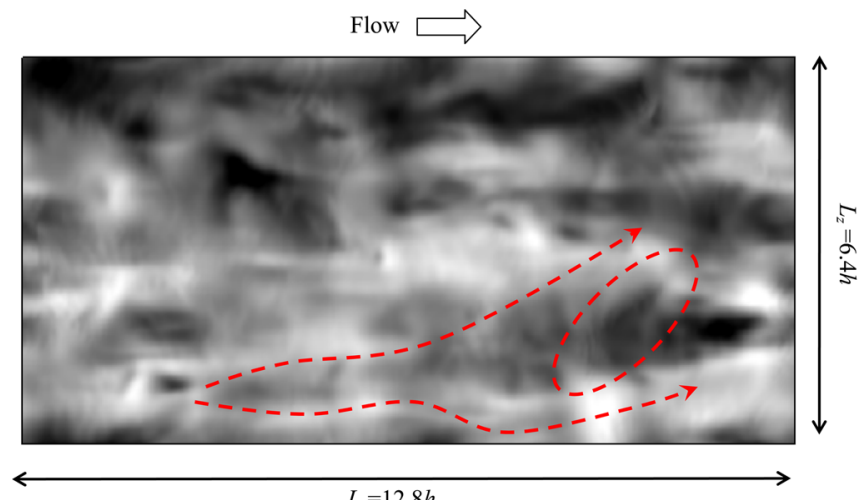

(a)

$L_{\mathrm{r}}=12.8 h$

Flow $\longrightarrow$

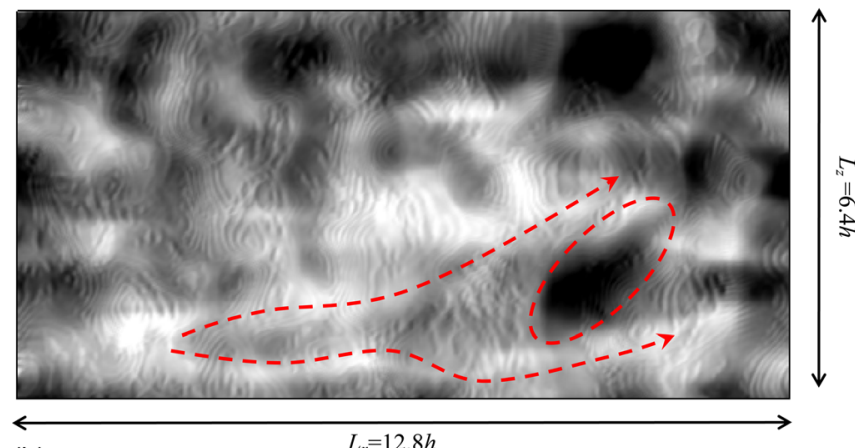

(b)

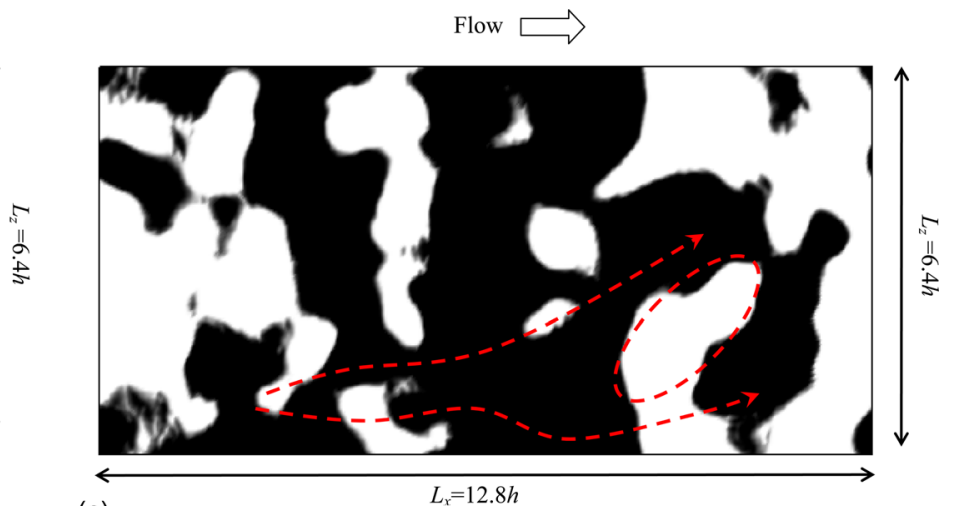

(c)

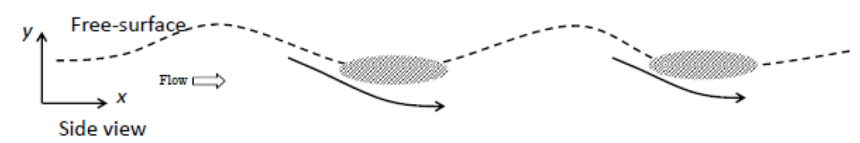

(d)

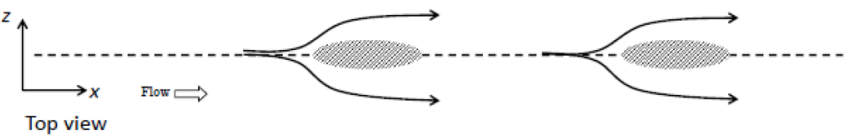

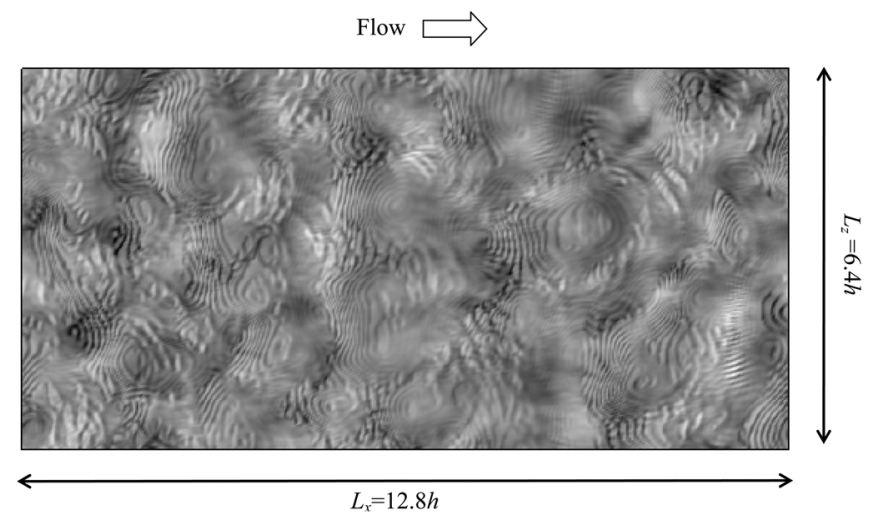

(e)

FIG. 8. (Color online) Flow visualization near free-surface, case 1: (a) streamwise turbulent velocity distributions, -1.9 (black) $<u^{+}<1.9$ (white), $y^{+}=138.5$, (b) streamwise turbulent velocity distributions, -1.9 (black) $<u^{+}<1.9$ (white), $y^{+}=149.7$, (c) VOF function distribution, 0.0 (white: air phase) $<F<1.0$ (black: water phase), $y^{+}=149.7$, (d) conceptual sketch of high-speed streaks and low-speed spots near free-surface, and (e) vertical turbulent velocity distributions, -1.9 (black) $<v^{+}<1.9$ (white), $y^{+}=149.7$.

\section{F. Water phase mean and turbulent statistics in high-Fr flow}

Figure 10 shows the near free-surface mean velocity profiles. Note that these mean velocities were averaged with a distinction made between the air and water phases, thus there are two mean velocity profiles of the air and water phases at the surface deformation areas, and the total mean velocity has the same profile as shown in Fig. 5(a). The water-phase mean velocity profile (plotted as $\bullet$ ) is almost constant; whereas, the velocity gradient of the air-phase profile (plotted as $\circ$ ) resembles a boundary layer profile. This suggests that the momentum transfer from the water phase to the air phase is active, while the momentum transfer from the air phase to the water phase is inactive. Total mean velocity profile (shown as a line) was as same one in Fig. 5(a).

Figure 11 shows the water-phase turbulent intensity profiles in case 1, 2, and 3. In the high-Fr case, turbulent intensities were averaged with a distinction made between the air and water phases, as in Fig. 10. In Fig. 11(a), the streamwise turbulent intensity in the high-Fr case (plotted as $\circ$ ) is equivalent to the parallel channel flow case (shown as a line), and the streamwise intensity in the low-Fr case (plotted as •) exceeded that in the other cases.

In Fig. 11(b), the wall-normal turbulent intensity in the low-Fr case (as plotted by $\mathbf{\Lambda}$ ) was suppressed by the presence of the rigid-lid free-surface, as pointed out in previous 

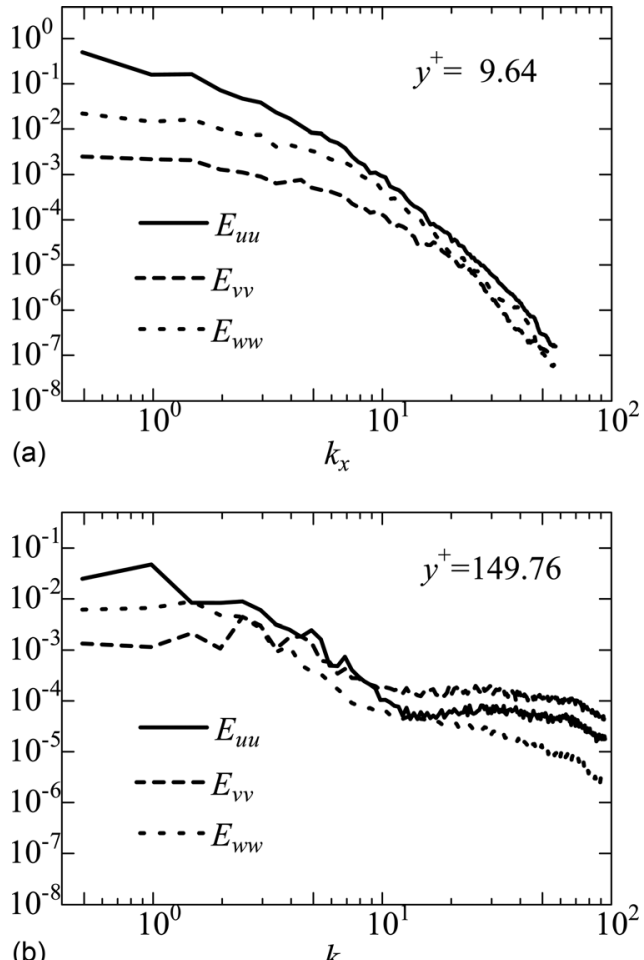

(b)

$k_{x}$

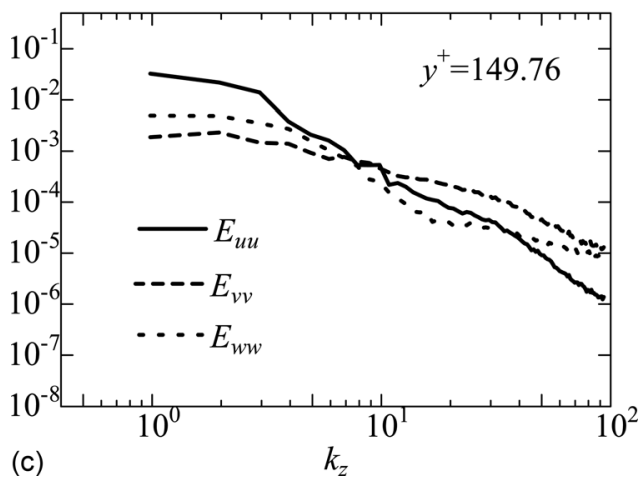

FIG. 9. Turbulent velocity energy spectrum profiles, case 1: near wall (streamwise), (b) at free-surface (streamwise), and (c) at free-surface (spanwise).

studies. ${ }^{3,5,9-17,19-21}$ On the other hand, the vertical turbulent intensity in the high-Fr case (plotted as $\triangle$ ) begins to increase up to the free-surface, and the wall-normal turbulence can be generated by the free-surface instabilities as

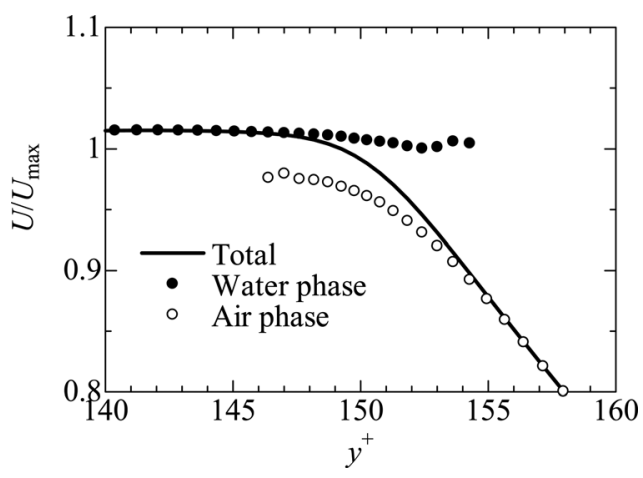

FIG. 10. Mean velocity profiles, near free-surface, case 1 .
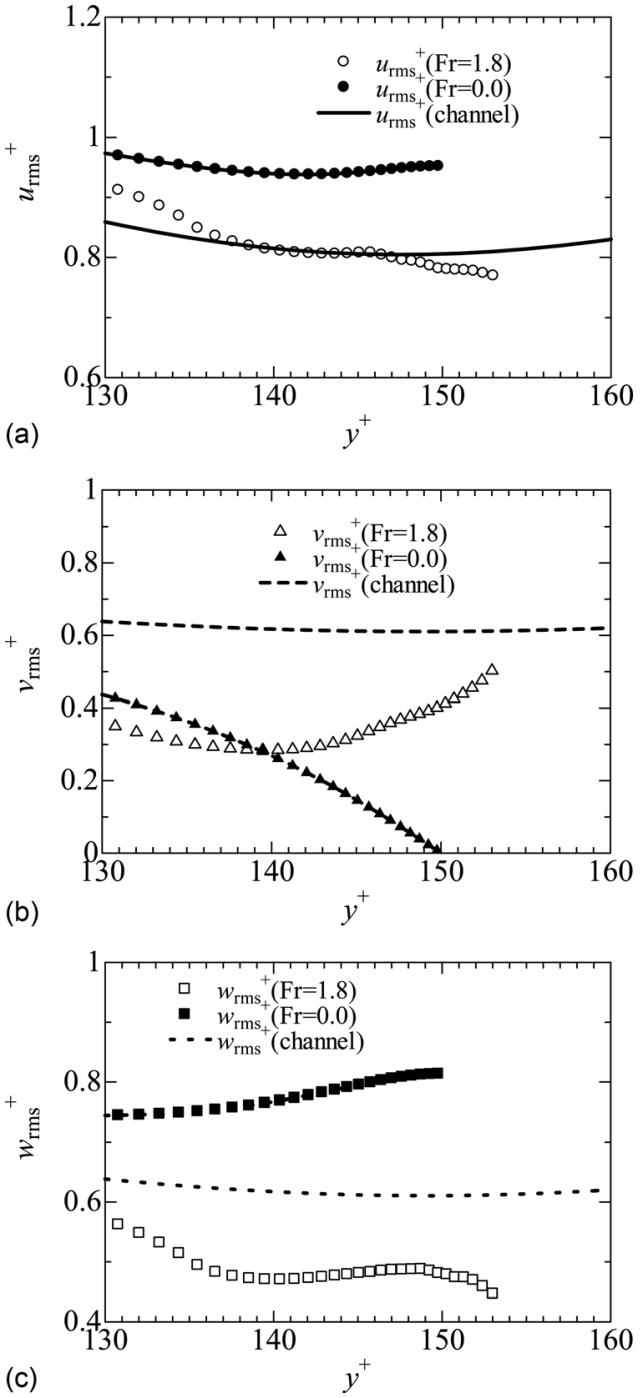

FIG. 11. Turbulent intensity profiles, near free-surface, case 1, 2, and 3: (a) streamwise turbulent intensity, (b) vertical turbulent intensity, and (c) spanwise turbulent intensity.

shown in Fig. 4(a). Consequently, the vertical confinement effect $^{3,5,9-17,19-21}$ at the free-surface is no longer present in the high-Fr case. The increase in the vertical turbulent intensity near the free-surface was also pointed out by the experimental measurement ${ }^{8}$ under the high-Fr condition $\mathrm{Fr}=1.5$.

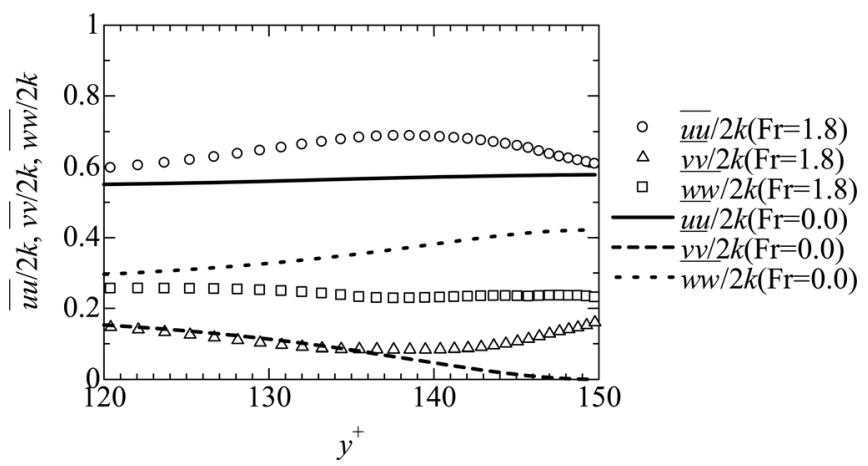

FIG. 12. Redistribution of turbulent kinetic energy, near free-surface, case 1 and 2 . 


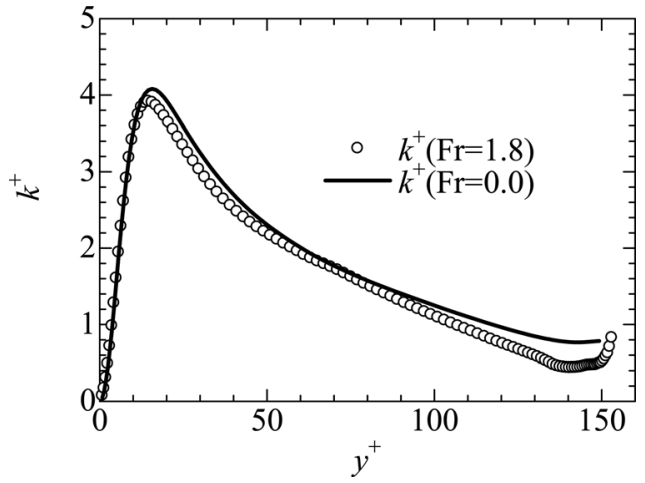

FIG. 13. Turbulent kinetic energy profiles, case 1 and 2.

The spanwise turbulent intensity in the low-Fr case (plotted as $\mathbf{\square}$ ), as shown in Fig. 11(c), was exceeded, and that in the high-Fr case (plotted as $\square$ ) is lower compared to the parallel channel flow case (shown as a dotted line).

Figure 12 shows the redistribution of near free-surface turbulent kinetic energy $(k)$ in case 1 and 2. Nezu and Nakayama ${ }^{28}$ and $\mathrm{Nezu}^{29}$ suggested the following experimental equations for the near free-surface redistribution of turbulent kinetic energy over a wide range of Froude number:

$$
\left.\begin{array}{l}
\overline{u u} / 2 k=0.55-0.05 / \exp (\mathrm{Fr}), \\
\overline{v v} / 2 k=0.17-0.17 / \exp (\mathrm{Fr}), \\
\overline{w w} / 2 k=0.28+0.22 / \exp (\mathrm{Fr}),
\end{array}\right\}
$$

where $\overline{u u}, \overline{v v}, \overline{w w},(2 k=\overline{u u}+\overline{v v}+\overline{w w})$ are streamwise, wall-normal, and spanwise Reynolds stress, respectively. Present DNS results in the high-Fr case (plotted as symbols) are consistent with the values of the above experimental equations for $\mathrm{Fr}=1.8$ of $\overline{u u} / 2 k=0.54, \overline{v v} / 2 k=0.14$, and $\overline{w w} / 2 k=0.31$.

Figure 13 shows the water-phase turbulent kinetic energy $(k)$ profiles in case 1 and 2. Turbulent kinetic energy is lower in the high-Fr case than that in the low-Fr case. One reason for this is that the turbulent kinetic energy may be transferred from the water phase to the air phase. In the highFr case, turbulent kinetic energy at the free-surface was compatible with the free-surface deformation scale $\left(k / g \approx h^{\prime}, h^{\prime}\right.$ : free-surface deformation scale, $h^{++}<6$ as shown in Fig. 3) but mean velocity scale at the free-surface was too large

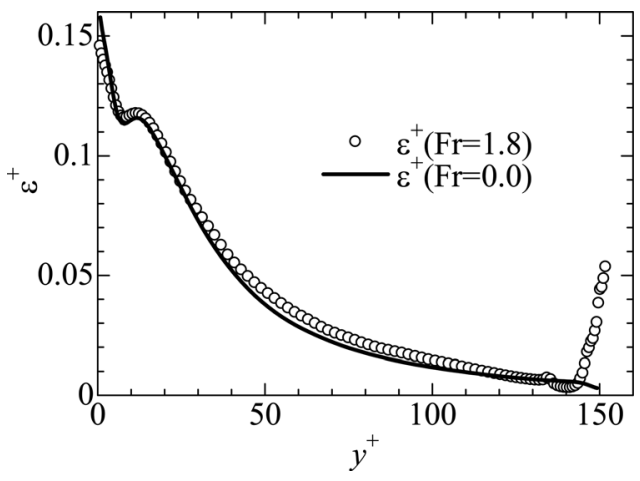

FIG. 14. Turbulent energy dissipation-rate profiles, case 1 and 2. compared with one $\left(U^{2} / 2 g \gg h^{\prime}\right)$. It suggested that surface deformation in this high-Fr flow would be scaled with turbulent kinetic energy at the free-surface.

Figure 14 shows the water-phase energy dissipation-rate profile. In the high-Fr case, the energy dissipation rate (plotted as $\bigcirc$ ) increases, but in the low-Fr case (shown as a line), it decreases slightly near the free-surface. This phenomenon was also observed by Nakayama and Nezu. ${ }^{8}$ However, Nakayama and Nezu estimated the energy dissipation-rate from the gradient of the velocity spectra because they did not measure it directly. Due to the increase of the energy dissipation-rate, Kolmogolov length $\left(l_{K}\right)$ was decreased toward the free-surface. Consequently, Kolmogolov length on the free-surface $\left(y^{+}=150\right)$ took 2 in wall-units and then, the horizontal maximum wave number normalized by Kolmogorov length $\left(k_{x} l_{K}\right)$ was estimated as 1.25 in case 1 . It was pointed out ${ }^{30}$ that the required grid resolution was more than $1 k_{x} l_{K}$ in the high-accuracy DNS. Therefore, the adequacy of the grid resolution in this DNS can be also confirmed.

Figure 15 shows the distribution of water-phase pressure-strain correlations in case 1,2, and 3. Pressure-strain correlations can be written as
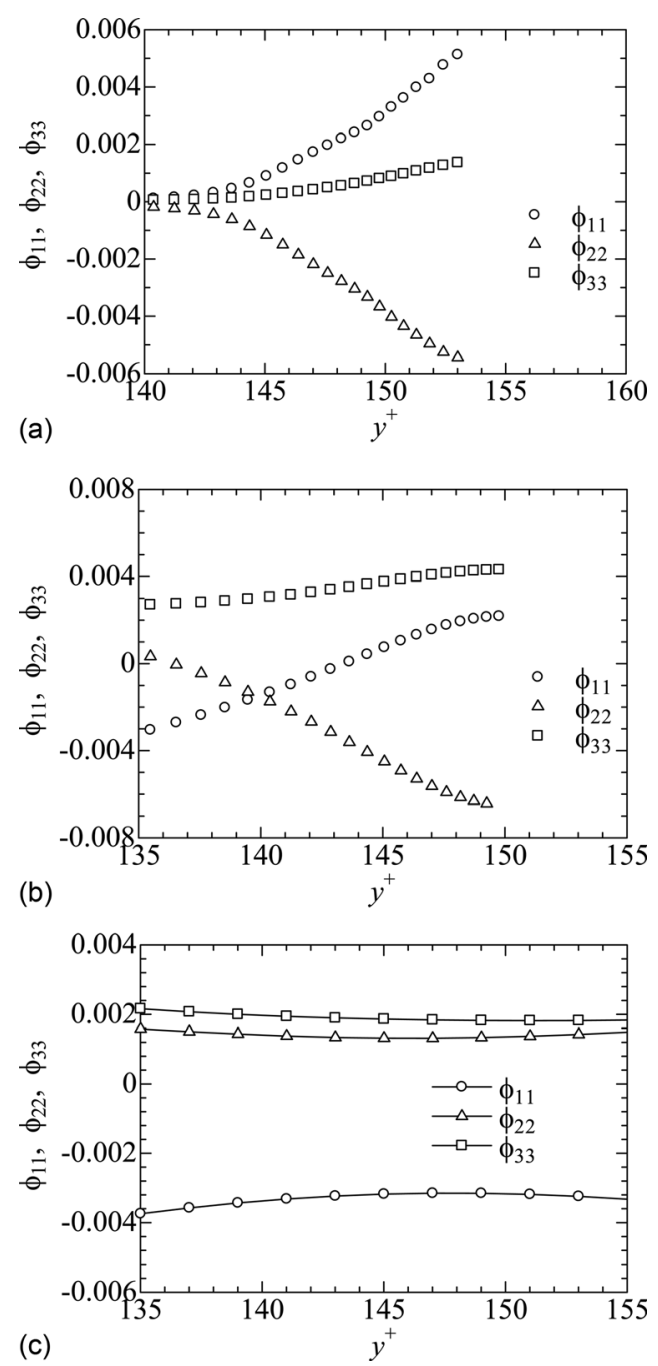

FIG. 15. Pressure-strain term profiles, case 1, 2, and 3: (a) case 1, (b) case 2 , and (c) case 3 . 


$$
\left.\begin{array}{l}
\phi_{11}=2 \frac{\bar{p} \frac{\partial u}{\partial x}}{\rho}, \\
\phi_{22}=2 \frac{\bar{p} \frac{\partial v}{\partial y}}{\rho \frac{p}{\rho} \frac{\partial w}{\partial z}}, \\
\phi_{33}=2 \frac{1}{\rho}
\end{array}\right\}
$$

where $\phi_{11}, \phi_{22}$, and $\phi_{33}$ denote the streamwise, vertical, and spanwise pressure-strain correlations, respectively. As shown in Fig. 15(a), the redistribution from the vertical pressurestrain correlation to the streamwise one is dominant, and little redistribution from vertical to spanwise correlation takes place in the high-Fr case. This causes the surface deformations to impact the wall-normal velocities at the free-surface, and the vertical turbulent energy beneath the free-surface is instantaneously transferred to the streamwise one, as shown in Figs. 8(b) and 8(e).

In the low-Fr case as shown in Fig. 15(b), the redistribution from the vertical pressure-strain correlation to others is dominant. In the low-Fr case, vertical velocity suppressed by the presence of the free-surface acting as a "weak wall" is mainly transferred to the spanwise one. Consequently, beneath the free-surface, the redistribution from the vertical component is dominant in both high-and low-Fr cases.

These pressure-strain correlation behaviors in the highand low-Fr cases agree well with the turbulent intensity profiles as shown in Fig. 11. In the parallel channel flow case shown in Fig. 15(c), the redistribution from the streamwise pressure-strain correlation to others is dominant near the channel center.

\section{CONCLUSIONS}

In this study, DNSs of high-Fr turbulent open-channel flow at a Froude number of 1.8 were conducted using the MARS method. DNS database such as the mean velocity, turbulent statistics, and surface fluctuations were established; near the free-surface, turbulent structures for high-Fr flow were investigated by comparing them with low-Fr flow. The following results were obtained:

(1) At the free-surface, two types of surface waves were observed in the high-Fr flow. One comprises large-scale gentle bumpy waves with a maximum wave height approximately corresponding to $4 \%$ of the water depth. The other comprises small-scale isotropic waves scaled with the turbulent kinetic energy at the free-surface.

(2) Near the free-surface in the high-Fr flow, wall-normal turbulent intensity and energy dissipation rate increased, and the turbulent kinetic energy decreased, as compared to the low-Fr flow.

(3) Behaviors of the turbulent statistics near the free-surface in the high-Fr flow agreed well with the LDA measurements. $8,25,26$

(4) Near the free-surface, the turbulent energy redistribution from the vertical pressure-strain correlation to the streamwise one was predominant in the high-Fr flow. This is because the surface deformations impact the wall-normal velocities at the free-surface. Consequently, beneath the free-surface, redistribution from the vertical velocity plays the primary role of turbulence transport in both the high- and the low-Fr flow.

\section{ACKNOWLEDGMENTS}

A part of the present DNS were conducted using SX-9 at the Cyber Science Center, Tohoku University, and the study was supported by the Global COE program "Energy Science in the Age of Global Warming" and a Grant-in-aid for Young Scientists (B), KAKENHI (21760156) MEXT, Japan.

${ }^{1}$ H. Nakagawa and I. Nezu, "Prediction of the contributions to the Reynolds stress from the bursting events in open-channel flows," J. Fluid Mech. 80, 99 (1977).

${ }^{2} \mathrm{H}$. Nakagawa and I. Nezu, "Structure of space-time correlations of bursting phenomena in an open-channel flow," J. Fluid Mech. 104, 1 (1981).

${ }^{3}$ S. Komori, H. Ueda, F. Ogino, and T. Mizushina, "Turbulent structure and transport mechanism at the free surface in an open-channel flow," Int. J. Heat Mass Transf. 25, 513 (1982).

${ }^{4}$ S. Komori, Y. Murakami, and H. Ueda, "The relationship between surface-renewal and bursting motions in an open-channel flow," J. Fluid Mech. 203, 103 (1989).

${ }^{5}$ I. Nezu and H. Nakagawa, "Turbulence in open-channel flows," in IAHRMonograph (Balkema Rotterdam, The Netherlands, 1993).

${ }^{6} \mathrm{M}$. Rashidi, "Burst-interface interactions in free surface turbulent flows," Phys. Fluids 9, 3485 (1997).

${ }^{7}$ S. Kumar, R. Gupta, and S. Banerjee, "An experimental investigation of the characteristics of free-surface turbulence in channel flow," Phys. Fluids 10, 437 (1998).

${ }^{8}$ T. Nakayama and I. Nezu, "Bursts near the free-surface in open-channel flows and their relationships with turbulence structures," J. Hydraulic, Coastal and Environmental Engineering JSCE, 635/II, 31 (1999) [in Japanese].

${ }^{9} \mathrm{~K}$. Lam and S. Banerjee, "On the condition of streak formation in a bounded turbulent flow,” Phys. Fluids A 4, 306 (1992).

${ }^{10}$ R. A. Handler, T. F. Swean, Jr., R. I. Leighton, and J. D. Swearingen, "Length scale and the energy balance for turbulence near a free surface," AIAA J. 31, 1998 (1993).

${ }^{11}$ S. Komori, R. Nagaosa, Y. Murakami, S. Chiba, K. Ishii, and K. Kuwahara, "Direct numerical simulation of three-dimensional openchannel flow with zero-shear gas-liquid interface," Phys. Fluids A 5(1), 115 (1993).

${ }^{12}$ D. M. Liu and G. Hetsroni, "Direct numerical simulation of a turbulent open channel flow with passive heat transfer," Int. J. Heat Mass Transf. 38, 3241 (1995).

${ }^{13}$ Y. Pan and S. Banerjee, "A numerical study of free-surface turbulence in channel flow," Phys. Fluids 7, 1649 (1995).

${ }^{14}$ V. Borue, S. A. Orszag, and I. Staroselsky, "Interaction of surface waves with turbulence: Direct numerical simulations of turbulent open-channel flow,” J. Fluid Mech. 286, 1 (1995).

${ }^{15}$ T. G. Thomas and J. R. Williams, "Turbulent simulation of open channel flow at low Reynolds number," Int. J. Heat Mass Transf. 38, 259 (1995).

${ }^{16} \mathrm{~B}$. Perot and P. Moin, "Shear-free boundary layers: Part 1. Physical insights into near-wall turbulence," J. Fluid Mech. 295, 199 (1995).

${ }^{17} \mathrm{~W}$. T. Tsai, "A numerical study of the evolution and structure of a turbulent shear layer under a free surface," J. Fluid Mech. 354, 239 (1998).

${ }^{18}$ D. T. Walker, R. I. Leighton, and L. O. Garza-Rios, "Shear-free turbulence near a flat free surface,” J. Fluid Mech. 320, 19 (1996).

${ }^{19}$ R. Nagaosa and T. Saito, "Turbulence structure and scalar transfer in stably stratified free-surface flows," AIChE. J. 43, 2393 (1997).

${ }^{20}$ R. Nagaosa, "Direct numerical simulation of vortex structures and turbulent scalar transfer across a free surface in a fully developed turbulence," Phy. Fluids 11, 1581 (1999).

${ }^{21}$ S. Yokojima, "Modeling and simulation of turbulent open channel flows emphasizing free-surface effects," Ph.D. thesis, Kobe University Japan, 2002.

${ }^{22}$ T. Kunugi, "MARS for multiphase calculation," Comput. Fluid Dyn. J. 9-1, 563 (2001).

${ }^{23}$ C. W. Hirt and D. B. Nichols, "Volume of fluid (VOF) method for the dynamics of free boundaries," J. Comput. Phys. 39, 201 (1981). 
${ }^{24}$ J. U. Brackbill, D. B. Kothe, and C. Zemach, "A continuum method for modeling surface tension," J. Comput. Phys. 100, 335 (1992).

${ }^{25}$ M. M. Rai and P. Moin, "Direct simulations of turbulent flow using finitedifference schemes," J. Comput. Phys. 96, 15 (1991).

${ }^{26}$ C. Canuto, M. Y. Hussaini, A. Quarteroni, and T. A. Zang, "Spectral Methods in Fluid Dynamics" (Springer-Verlag, New York, 1988).

${ }^{27}$ M. J. Lee, J. Kim, and P. Moin, "Structure of turbulence at high shear rate," J. Fluid Mech. 216, 561 (1990).
${ }^{28}$ I. Nezu and T. Nakayama, "Mutual interaction between bursts and boils very near the free-surface of open-channel flows." in Environmental hydraulics, edited by J. H. W. Lee et al., (Balkema Rotterdam, The Netherlands, 1998), p. 297.

${ }^{29}$ I. Nezu, "Open-channel flow turbulence and its research prospect in the 21st century,” J. Hydraulic Eng. 131229 (2005).

${ }^{30} \mathrm{~V}$. Eswaran and S. B. Pope, "An examination of forcing in direct numerical simulations of turbulence," Comput. Fluids 16, 258 (1988). 